\title{
Trends and advances of teleconsultation in developing countries
}

\author{
Bartolo $\mathrm{M}^{1 *}$, Ferrari $\mathrm{F}^{2}$, Erba $\mathrm{F}^{3}$, Petrolati $\mathrm{S}^{4}$, Leone $\mathrm{M}^{5}$ and Enrico Fraschetti ${ }^{6}$ \\ ${ }^{1}$ GHT General Secretary; Director of Telemedicine Unit, Ospedale San Giovanni, Rome, Italy \\ ${ }^{2}$ Resident in Radiodiagnostic, University La Sapienza, Rome, Italy \\ ${ }^{3}$ DREAM Program. Comunità di Sant'Egidio, Rome, Italy \\ ${ }^{4}$ Ospedale San Camillo, Cardiovascular Departement, Rome, Italy \\ ${ }^{5}$ IRCCS Istituto C. Besta, Neurology. Milan, Italy \\ ${ }^{6} \mathrm{GHT}$ software developing and IT consulatancy service
}

Global Health Telemedicine (GHT) is a non-profit organization created in September 2013 in charge of offering specialized medical tele-consultation to Sub-Saharan Africa medical centers.

Through a particular software enhanced by BS-Innova ${ }^{\mathrm{TM}}$, the local doctor can live submit a request to the GHT volunteer specialist attaching clinical question, triage, anamnesis, previous medical examinations and laboratory exams, essential instrumental tests, pictures and videos. Simultaneously, through an SMS, the italian specialist receives the tele-consultation request, inviting him to log in the platform in order to proceed to reply. The requesting doctor immediately receive the required advice.

Currently the active branches are sixteen: cardiology, neurology, ophtalmology, internal medecine, radiodiagnostic, infectious diseases, general surgery, nutrition, ophtalmology, hematology, orthopedics, urology, endocrinology, hepatology, vascular diseases and dermatology. Twenty-one GHT centers opened in Central and Southern Africa: four in Mozambique and Tanzania, three in Malawi, two in Kenya and one in Angola, Camerun, Centrafrican Republic, Democratic Republic of Congo, Guinea Conakry, Nigeria, Swaziland and Togo. Each GHT center supports already exisiting medical centers and is equipped with basic instrumentation, as electrocardiograph, pulse oximeter, back light panels, scanner for X-ray film and internet acces for sending tele-consultation requests. In thirty months activity we provided more than 2500 (2569) tele-consultations, receiving an average of 30 weekly requests. Cardiology is the most required branch with 2000 teleconsultations (77\%), followed by infectious diseases with $230(9 \%)$ and radiology with 105 (4\%). Most of these came from Arusha-Tanzania (1257 requests, 49\%) and from Beira-Mozambique (750, 29\%) wich are the first GHT opened centers. The GHT volunteer doctors involved in the project are fifty, working in different italian cities and contexts, and the average response time is about 17 hours.

Our experience clearly shows that the increase in life expectancy in developing countries in which we are involved, caused the increase of chronic, and before unknown, degenerative diseases, as the cardiovascular and the metabolic one. Some of these diseases need a local outpatient tratment, and receiving an expert second opinion, as a telemedical one, would positively impact the patient outcoming.
Copyright: (C2016 Bartolo M. This is an open-access article distributed under the terms of the Creative Commons Attribution License, which permits unrestricted use, distribution, and reproduction in any medium, provided the original author and source are credited.
Correspondence to: Fabio Ferrari, Resident in Radiodiagnostic, University La Sapienza. Rome, Italy, E-mail: fabioferrari84@yahoo.it

Received: July 06, 2016; Accepted: July 16, 2016; Published: July 19, 2016 